\title{
Observatório como instrumento de prospectiva estratégica para as Instituições de Ciência e Tecnologia (ICTs)
}

\author{
Observatory as a strategic prospective instrument for Science and Technology \\ Institutions (ICTs)
}

\author{
Observatoire comme instrument de prospective stratégique pour les \\ Institutions de la Science et de la Technologie (ICTs)
Observatorio como instrumento de prospectiva estratégica para las Instituciones de Ciencia y Tecnología (ICTS)

\author{
Nádia Solange Schmidt ${ }^{1}$ \\ Christian Luiz da Silva²
}

Recebido em 16/08/2017; revisado e aprovado em 01/10/2017; aceito em 01/10/2017

DOI: http://dx.doi.org/10.20435/inter.v19i2.1689

\begin{abstract}
Resumo: Este artigo a presenta um modelo de Observatório para as Instituições de Ciência e Tecnologia (ICTS), que atuam junto às cadeias agropecuárias. O objetivo é utilizar um processo sistemático para vislumbrar a médio e longo prazo, o futuro da ciência e da tecnologia, identificando áreas estratégias de pesquisa e de tecnologias emergentes que se relacionam a benefícios econômicos e sociais. A principal forma de atuação do modelo proposto é a interação entre os pesquisadores e os atores da cadeia. O modelo foi avaliado e aprovado por 96,7\%dos participantes da pesquisa.
\end{abstract}

Palavras-chave: prospectiva tecnológica; observatório; Instituições de Ciência e Tecnologia; cadeias produtivas.

Abstract: This article presents a model of an Observatory for Science and Technology Institutions (ICT's ), which work together with the agricultural chains. The objective is to use a systematic process to antecipate the future of science and technology in the medium and long term, identifying areas of research strategies and emerging technologies that relate to economic and social benefits. The main form of action of the proposed model is the interaction between researchers and actors in the chain. The model was evaluated and approved by $96.7 \%$ of the study participants.

Keywords: technological foresight; observatory; Science and Technology Institutions; production chains.

Résumé: Cet article présente un modèle d’Observatoire pour les Institutions Scientifiques et Technologiques (ICT's ), qui coopère avec les chaînes agricoles. L'objectif est d'utiliser un processus systématique pour anticiper l'avenir de la science et de la technologie à moyen et à long terme, en identifiant les domaines de stratégies de recherche et les technologies émergentes qui concernent les avantages économiques et sociaux. La forme d'action principale du modèle proposé est l'interaction entre les chercheurs et les acteurs de la chaîne. Le modèle a été évalué et approuvé par 96,7\% des participants à l'étude.

Mots-clés: prospective technologique; observatoire; Institutions Scientifiques et Technologiques; chaînes de production;

Resumen: Este artículo presenta un modelo de Observatorio para Instituciones de Ciencia y Tecnología (ICTS), que actúan junto a cadenas agropecuarias. El objetivo es el uso de un proceso sistemático para la búsqueda de un medio y largo plazo, el futuro de la ciencia y la tecnología, identificando las áreas de investigación y de tecnologías emergentes que se relacionan con los beneficios económicos y sociales. Una forma principal de actuación del modelo propuesto es una interrelación entre los investigadores y los atores de la cadena. O modelo fue aprobado y aprobado por 96,7\% dos participantes de la investigación.

Palabras clave: prospectiva tecnológica; observatorio; Instituciones de Ciencia y Tecnología; cadenas productivas.

\footnotetext{
${ }^{1}$ Empresa Brasileira de Pesquisa Agropecuária (EMBRAPA), Concórdia, Santa Catarina, Brasil.

${ }^{2}$ Universidade Tecnológica Federal do Paraná (UTFPR), Curitiba, Paraná, Brasil.
} 


\section{INTRODUÇÃO E OBJETIVOS}

De acordo com Boehm e Hogam (2013), a pesquisa colaborativa entre ICTs e empresas tem forte impacto sobre o sucesso da transferência de conhecimento, por isso é importante estudar a interação ciência-mercado para fins científicos e de comercialização do conhecimento.

Amparo, Ribeiro e Guarieiro (2012) afirmam que na área tecnológica - primordial para o desenvolvimento do país, a necessidade de ferramentas prospectivas é indispensável para orientar o futuro. A inovação tem três etapas distintas: pesquisa e desenvolvimento e inovação (PD\&I) - está em grande parte, sob a responsabilidade das ICTs, pois o setor privado brasileiro não tem tradição em investir em pesquisa; Produção- ocorre mais comumente nas empresas e se inicia com os resultados da etapa anterior, somadas as tendências de mercado e à expectativa de lucros; e comercialização que se traduz em divisas e informações que realimentarão o processo, fechando o ciclo de geração de conhecimento e inovação Cordeiro (2003).

Devido ao alto custo e temporalidades das pesquisas científicas e tecnológicas, observa-se uma preocupação crescente dos governos em apoiar e estabelecer estreita parceria com o setor produtivo, considerado por eles sustentáculo da vantagem competitiva nacional (PORTER, 2004), para dividir custos e resultados e tornar o processo de PD\&I mais ágil e efetivo. Estudo desenvolvido por Soh e Subramanian (2014) revelam que as empresas com maior capacidade interna de PD\&I ganham mais benefícios em atividades colaborativas com as ICTs públicas e vice-versa. Entretanto a sobreposição de recursos e conhecimentos entre empresas e ICTs pode levar a custos de redundância e coordenação do conhecimento.

Soh e Subramanian (2014) afirmam que, pela colaboração público-privada na transformação de descobertas científicas em tecnologias comerciais, as ICTs exploram ativamente suas descobertas e promovem a comercialização de tecnologia (LAVIE; DRORI, 2012), enquanto que para as empresas a colaboração é imperativa não só para acessar e alavancar recursos valiosos, como cientistas e instalações de pesquisa de ponta, mas também para explorar conhecimento científico e descobertas inovadoras emergentes (AUDRETSCH; LEYDEN; LINK, 2012; SUBRAMANIAN; LIM; SOH, 2013).

No Brasil, o agronegócio é o principal alicerce socioeconômico. A competitividade dos produtos é resultado do uso de tecnologias avançadas, sendo que a maior parte é importada de países desenvolvidos, uma vez que, no Brasil, a pesquisa é essencialmente básica, e o governo não dispõe de recursos para passar da fase fundamental para a produção em escala (CORDEIRO, 2003). Essas características presentes na CT\&I brasileira, deixam clara a necessidade de ampliar o uso de ferramentas prospectivas para buscar o desenvolvimento e a independência tecnológica.

Este estudo propõe um modelo de Observatório, voltado para o monitoramento e identificação de tendências tecnológicas nas cadeias produtivas do agronegócio, tendo como objeto de estudo a cadeia produtiva de frangos de corte. Todavia esse modelo pode ser replicado para as demais cadeias produtivas. A motivação principal paira na necessidade identificar e antecipar as demandas para criar soluções efetivas e promover a interação entre o setor público e privado.

O artigo está sumarizado em 10 seções, iniciando-se com a introdução e objetivos, seguido da apresentação da metodologia. Em sequência, são tratadas questões referentes a Ciência, tecnologia e Inovação tecnológica. A seção quatro aborda alguns conceitos sobre prospectiva tecnológica e seus usos. Na seção cinco, há informações resumidas sobre a Embrapa e a seção seis, sobre a cadeia produtiva de frangos de corte. A seção sete aborda Observatórios e seu uso 
como ferramenta prospectiva. O modelo de observatório está descrito na seção oito, seguido das conclusões e das referências.

\section{METODOLOGIA}

A metodologia deste trabalho envolveu três fases distintas:

1) Diagnóstico sobre a atuação da Embrapa e a adoção das tecnologias por ela desenvolvidas junto à cadeia produtiva de frangos de corte:

2) Desenvolvimento do modelo: foram feitas pesquisa bibliográficas e documental e análise dos dados obtidos nos questionários enviados aos agentes da cadeia, pesquisadores e profissionais de Transferência de Tecnologia da Embrapa Suínos e Aves e da Agricultural Research Service (ARS), nos Estados Unidos. Para pesquisa bibliográfica, utilizou-se a base de dados da Web Science, Scopus, Science Direct e Scielo. Foram entrevistados um total de 236 atores.

3) Validação do modelo: O modelo foi validado por meio de questionários semiestruturados enviados por meio do software Lime Survey para os mesmos agentes da cadeia que participaram da pesquisa sobre o desenvolvimento do modelo. O modelo foi validado por 167 agentes, dos quais $96,77 \%$ aprovaram o modelo proposto.

Para a seleção das empresas (e agentes) participantes, utilizou-se o Guia Gessulli de Avicultura Industrial ${ }^{3}$ e indicações de pesquisadores da unidade. Os objetivos da pesquisa, descrição dos entrevistados e critério de seleção encontram-se descritos, de forma sucinta, no quadro 1.

\begin{tabular}{|l|l|l|}
\hline \multicolumn{1}{|c|}{ Objetivo das entrevistas } & \multicolumn{1}{|c|}{ Entrevistados } & \multicolumn{1}{|c|}{ Critério de seleção } \\
\hline $\begin{array}{l}\text { Avaliar as tecnologias geradas pela } \\
\text { Embrapa Suínos e Aves }\end{array}$ & Pesquisadores da instituição & Cargo e Conhecimento \\
\hline $\begin{array}{l}\text { Obter um diagnóstico sobre a atuação da } \\
\text { Embrapa Suínos e Aves na cadeia }\end{array}$ & $\begin{array}{l}\text { Pesquisadores da Embrapa e agentes } \\
\text { da cadeia }\end{array}$ & Cargo e conhecimento \\
\hline $\begin{array}{l}\text { Desenvolver um modelo de prospectiva } \\
\text { estratégica para as instituições de C\&T }\end{array}$ & $\begin{array}{l}\text { Pesquisadores e profissionais de TT da } \\
\text { Embrapa, da ARS e agentes da cadeia. }\end{array}$ & Cargo e conhecimento \\
\hline Validação do modelo proposto & Agentes da cadeia & Cargo e conhecimento \\
\hline
\end{tabular}

Quadro 1 - Objetivo das entrevistas, entrevistados e critérios de seleção

Fonte: Dados da pesquisa.

A população que compunha os agentes que atuam na cadeia é elevada, sendo necessário caracterizar uma amostra (MARCONI; LAKATOS, 2007). Utilizou-se, então, a amostragem aleatória simples, na qual todos os elementos têm a mesma probabilidade de serem selecionados. O erro amostral atribuído para o cálculo foi 5\%, com um nível de confiança de 95\% para uma população de 190 pessoas. Nesse caso, o número da amostra desejada é de 128 . O número de respondentes foi de 167, superior ao número da amostra desejada. As análises estatísticas foram realizadas por meio dos softwares IBM SPSS (Statistical Package for the Social Sciences) v. 21, IBM SPSS Amos v. 21.0.0 e Microsoft Excel, na etapa de avaliação dos dados.

\footnotetext{
${ }^{3}$ Disponível em: <http://www.guiagessulli.com.br/>.
} 


\section{CIÊNCIA, TECNOLOGIA \& INOVAÇÃO TECNOLÓGICA}

As duas entidades, ciência e tecnologia, não têm a mesma natureza e possuem especificidades próprias, contudo há laços significativos entre as duas entidades. A ciência, orientada pelo desejo de conhecer e explicar, e a tecnologia, orientada pelo desejo de controlar e de modificar, estão profundamente ligadas, apesar de cada uma delas ter desenvolvido modos de operar diferenciados. A ciência evoluiu, quase só, no sentido da abstração e da teoria, enquanto a atividade tecnológica se desenvolveu para a concretização de algo e de uma forte ação prática (SANTOS, 1989).

\subsection{Ciência}

De acordo com Vasconcellos (2008), a ciência tem duas grandes vertentes: a primeira, relacionada à invenção de instrumentos e implementos para superar os desafios; e a segunda representa a forma pela qual o homem procura compreender e explicar o universo que o envolve, à luz das suas crenças. A primeira vertente pode receber o nome de tecnologia, pois se torna ciência aplicada; a segunda vertente, que nos tempos históricos se desenvolveu como pura busca de conhecimento, é a ciência básica (VASCONCELLOS, 2008).

Segundo Schwartzman (2002), no Brasil, as atividades de pesquisa não se constituem de forma explícita, com recursos e procedimentos claros de contratação e acompanhamento de projetos, mas ocorrem pela utilização de recursos que podem ser muito significativos para os pesquisadores, mas insignificantes em relação às atividades centrais dos governos. Os possíveis vínculos e tensões entre a ciência básica e a ciência aplicada têm sido objeto de constante preocupação. O autor cita um estudo que, feito por Eduardo Krieger e Fernando Galembeck sobre a pesquisa brasileira, em meados dos anos 1990, concluiu que existe um grande distanciamento entre o mundo da pesquisa e o mundo empresarial devido, principalmente, ao permanente conflito de interesses entre os defensores da "pesquisa básica" e da "pesquisa aplicada", em que se ignora a grande interdependência e necessidade de ambas e a falta de vínculos entre pesquisadores e profissionais de empresas e existência de muitos argumentos de rejeição mútua entre ambos.

Os cientistas justificam suas verbas pela convicção de que seu trabalho é sempre útil e importante e, nesse sentido, sempre aplicado. O que muitas vezes não se percebe é que a transformação de conhecimentos "básicos" ou "aplicados" em resultados efetivos depende de arranjos institucionais que geralmente, não existem nos ambientes públicos (SCHWARTZMANN, 2002).

\subsection{Tecnologia}

Embora o termo 'tecnologia' seja familiar em nosso cotidiano, sua definição não é tão evidente, pois a fronteira com a ciência nem sempre é clara. Nelson (2004) define tecnologia como sendo a variedade de técnicas produtivas voltadas às necessidades e que foram desenvolvidas ao longo dos anos pelas pessoas, que vão desde designs sofisticados de produtos a procedimentos aplicáveis em diversos setores.

De forma semelhante, Dosi (1982) define tecnologia como um conjunto de peças de conhecimento, tanto prático como teórico, know-how, métodos, procedimentos, experiências de sucessos e também de falhas. Tecnologia, nesse ponto de vista, inclui apercepção de um conjunto limitado de possíveis alternativas tecnológicas e de futuros desenvolvimentos teóricos. 
A tecnologia deve criar a capacidade humana de "fazer", e deve ser utilizada para pensar produtos novos e úteis (DEVORE, 1987). Resultados comuns ou traços da nova ciência (tecnologia) são a previsibilidade, replicação, confiabilidade, otimização e eficiência das operações do sistema baseados em modelos teóricos (DEVORE, 1987).

A expressão 'ciência e tecnologia' designa uma unidade e, entre o público em geral, a ciência tende a ser valorizada por suas contribuições à tecnologia. Em contrapartida, a tradição da ciência moderna considera a tecnologia como um mero subproduto e valoriza a ciência pelo entendimento do mundo que ela proporciona. Essa tradição interpreta a ciência como "algo que existe em função da apreensão da estrutura causal do mundo e da sintetização de suas possibilidades, não por causa de seus subprodutos tecnológicos.

Dizer que a nova tecnologia promove uma nova ciência é tão verdadeiro quanto afirmar o contrário. A questão é que o aumento da ciência baseada em tecnologia conduziu a uma dramática mudança na natureza das pessoas e das instituições envolvidas no avanço tecnológico; não se pode mais fazer ciência e tecnologia sem profissionais preparados para isso (VASCONCELLOS, 2008).

\subsection{Inovação tecnológica}

Freeman (1982) chama a atenção para três equívocos conceituais na compreensão da inovação tecnológica: reducionismo (considerar inovação apenas a de base tecnológica), encantamento (considerar inovação apenas a espetacular) e descaracterização (relaxar o requisito de mudança tecnológica desta inovação).

Segundo Vilha (2010), a inovação tecnológica é baseada em atividades realizadas na empresa com uso de P\&D, contratação de mão de obra qualificada, aquisição/licenciamento de tecnologias, investimento produtivo e ações de marketing. Paralelamente, a empresa interage e combina seus conhecimentos internos com fontes externas de informação e de cooperação tecnológica. Esse processo é permeado por leis e políticas científicas e tecnológicas regidas pelo Governo e conta também com o aparato de instituições para financiar o processo inovativo (VILHA, 2010).

Shapira e Goldenberg (2007) estudaram os modelos clássicos de desenvolvimento tecnológico e afirmam que esses modelos sugeriam um percurso linear da pesquisa básica e desenvolvimento, para a comercialização e a adoção da tecnologia, ou vice-versa.

O modelo linear surgido a partir do fim da 2a - Guerra Mundial dominou o pensamento sobre inovação em C\&T por cerca de três décadas. O modelo interativo (chain-link model) foi inicialmente proposto por Kline e Rosenberg (1986), e logo se tornou o modelo que se contrapôs ao modelo linear.

Campos (2006) destaca que o modelo linear parte do erro fundamental de considerar o fluxo de informações a partir de um processo unidirecional e chega à etapa comercial sem que haja o retorno de informações para a pesquisa. Outra perspectiva, chamada demand pull ou modelo linear reverso, considerou que a demanda de mercado induz o processo de inovação (SCMOOKLER, 1962 apud CAMPOS, 2006).

Entretanto a hipótese de que apenas a demanda de mercado direciona o processo de inovação não foi suficiente para explicar a direção e o ritmo do processo de inovação. A demanda de mercado é vista como uma condição necessária para uma mudança tecnológica, mas não como uma condição suficiente. Apesar das limitações dessa perspectiva, seus estudos contribuíram para o reconhecimento do fator "demanda de mercado" como importante no processo inovativo. 


\section{PROSPECTIVA TECNOLÓGICA: CONCEITUALIZAÇÃO E USOS}

Mayerhoff (2008) se refere à prospectiva como o estudo do futuro para o desenvolvimento de uma atitude estratégica na criação de um futuro desejável. Para Ben Martin (1995), a prospectiva é um processo que se ocupa de forma sistemática de vislumbrar, a longo prazo, o futuro da ciência e da tecnologia, da economia e da sociedade, para identificar áreas estratégias de pesquisa e de tecnologias emergentes que se relacionam a benefícios econômicos e sociais.

Para Godet e Durance (2011), a prospectiva deve ter por objetivo tornar a ação eficaz. Trata-se de uma ciência da prática que não consiste em observar o futuro a partir do presente, mas em observar o presente a partir do futuro, o que implica a escolha de um futuro entre os muitos futuros possíveis, evitando perder tempo com falsos problemas ou questões ultrapassadas.

A atividade prospectiva está estreitamente vinculada ao planejamento, e sua importância reside na tentativa de determinar os acontecimentos futuros, no aumento da capacidade de inovar por meio de um processo organizado e na criação de consenso sobre as tendências (SANZ; ANTÓN, CABELLO, 2010). A prospectiva e a estratégia são indissociáveis, e convém separar o tempo da elaboração e avaliação das opções estratégicas possíveis para a organização se preparar para as mudanças esperadas e provocar as mudanças desejáveis (GODET; DURANCE, 2011).

Canongia, Pereira e Antunes (2006) afirmam que, nos países em desenvolvimento, o desafio é construir um ambiente favorável para expandir os setores, aumentar a competitividade e desenvolver e aprimorar novas tecnologias, antecipando oportunidades e ameaças. Pensar de forma global e agir local tem sido uma exigência constante, impondo mudanças rápidas de estratégias.

Nesse contexto, estudos prospectivos em setores intensivos em PD\&I são prioritários para alcançar competitividade e o desenvolvimento econômico, social e tecnológico. A prospectiva nas ICTs deve ser desmistificada, devendo tornar-se uma ferramenta rotineira de apoio à tomada de decisões para melhorar a gestão da inovação e ampliar a visão dos gargalos tecnológicos e das oportunidades a eles associadas.

Para Godet e Durance (2011), a base de toda prospectiva compreende a realização de um diagnóstico retrospectivo e estratégico a longo prazo, a análise das tendências, a detecção dos sinais fracos e a identificação das rupturas temidas ou desejadas para o futuro e as suas consequências. As atividades prospectivas devem envolver o maior número possível de atores para legitimar a decisão, se mostrando valiosa no planejamento de pesquisas científicas, já que esta poderá otimizar o uso e gestão de recursos, evitando desperdícios financeiros, materiais e de tempo na identificação de oportunidades e ameaças, além de facilitar a gestão da inovação e ampliar a visão dos gargalos tecnológicos e das oportunidades a eles associadas (QUINTELLA et al., 2011).

\section{A EMBRAPA}

A Empresa Brasileira de Pesquisa Agropecuária-Embrapa foi criada na década de 1970, com objetivo de enfrentar os problemas de abastecimento, expandir e diversificar as exportações (MARRA; SOUZA; ALVES, 2013). A Embrapa é parte de um grande complexo de Ciência, Tecnologia e Inovação (CT\&I), que abastece o agronegócio com tecnologias e é abastecido por ele com problemas e demandas a serem solucionadas.

As pesquisas realizadas pela instituição são financiadas com recursos públicos. A Embrapa é maior e mais importante instituição pública de pesquisa brasileira. Atua por intermédio 46 unidades de descentralizadas de pesquisa e 16 escritórios de negócio, distribuídos em todo território nacional. Além disso, possui quatro Laboratórios virtuais (EUA, Europa, China e Coreia do 
Sul) e três escritórios internacionais (América Latina e África). Uma das unidades descentralizadas de pesquisa é a Embrapa Suínos e Aves, criada em 1975, para oferecer soluções tecnológicas para apoiar o desenvolvimento e competitividade das cadeias avícola e suinícolas brasileiras.

A Embrapa, assim como a unidade estudada, utiliza o modelo Linear de pesquisa, no qual o desenvolvimento, a produção e a comercialização de novas tecnologias são vistos como uma sequência de tempo bem definida, que se origina nas atividades de pesquisa básica. A adoção de modelo resulta na baixa interação com os usuários da tecnologia e dificulta a identificação de demandas de pesquisa. Como resultado, as tecnologias desenvolvidas nem sempre atendem as reais necessidades dos usuários, já existem no mercado ou estão ultrapassadas, tornando-se "pesquisas de prateleiras" (BASSI, 2015). Esse fato é percebido pelos pesquisadores da instituição, que apontam a necessidade de mecanismos prospectivos que permitam identificar demandas e tendências futuras, e assim, desenvolver pesquisas que resolvam os problemas da cadeia (BASSI; SILVA; FIGUEIREDO, 2015).

\section{A CADEIA PRODUTIVA DE FRANGOS DE CORTE BRASILEIRA}

A cadeia produtiva de frangos brasileira é considerada uma atividade econômica internacionalizada e com uso intensivo de alta tecnologia. Segundo Costa, Garcia e Brene (2015), o dinamismo da cadeia aliado às mudanças nas formas de produção, industrialização, comercialização e consumo the conferiram competitividade, expressa por impressionantes ganhos de produtividade, resultando na queda progressiva do preço da carne.

De acordo com a ABPA (2016), a avicultura emprega cerca de 3,56 de brasileiros (5\% da população ocupada no país). Em 2015, o Brasil passou a ocupar o segundo lugar na produção mundial de carne de frango, com 13,14 milhões de toneladas, atrás apenas dos Estados Unidos. E, desde 2010, ocupa a liderança mundial na exportação de carne de frango (ABPA, 2016).

Jesus Junior et al. (2007) afirmam que essa cadeia apresenta uma das trajetórias mais importantes dentre as cadeias produtivas agroindustriais brasileiras, marcada por constantes evoluções técnicas e tecnológicas, que resultaram na conquista do mercado interno e externo, superando os principais fornecedores avícolas mundiais. Os principais elos inovadores, de acordo com o autor, estão localizados no elo de produção.

Por se tratar de uma cadeia altamente competitiva, com uso de tecnologias avançadas e um mercado dinâmico, os problemas, quando se apresentam, exigem rapidez na busca de soluções. A morosidade e a burocracia, inerentes às instituições públicas brasileiras, são um fator impeditivo para as ações de cooperação, levando a cadeia buscar parceiros mais ágeis sem os entraves jurídicos presentes nas instituições públicas (BASSI; SILVA; SANTOYO, 2013/2014).

\section{OBSERVATÓRIOS: CONCEITUAÇÃO E USO COMO FERRAMENTA PROSPECTIVA}

Os observatórios em C\&T foram criados com a tarefa de gerir a informação para transformá-la em conhecimento útil aos diferentes atores (DE LA VEGA, 2007). Sua criação está relacionada a seu caráter inovador e capacidade de produzir informações com valor agregado, por contar com a participação de várias instituições. De acordo com o autor, a cultura de cada país interfere no desempenho deste. Quanto mais desenvolvido, maior e melhor o tratamento dos dados, a produção uso e valorização do conhecimento gerado por parte das instituições, que são as principais fontes de informações e as que mais se beneficiam das informações geradas. 


\subsection{Conceituação}

Não há muitas definições de observatório na literatura. Para Testa (2002), observatório é um sistema organizado e estruturado de coleta, descoberta e análise de informações sobre o ambiente de um determinado setor de atuação. O sistema da Federação das Indústrias do Paraná (FIEP, 2015), por sua vez, define o observatório como um dispositivo de observação criado por um ou vários organismos, para acompanhar a evolução de um fenômeno, de um domínio ou de um tema estratégico, no tempo e no espaço.

Enjunto (2008) classifica observatório como uma instância criada por um coletivo, para acompanhar a evolução de um fenômeno, geralmente social, a partir de um ponto de vista. Ele indicou que existem diferentes tipos de observatórios; alguns promovido pela administração, outras organizações sociais ou empresas; com cobertura nacional, regional ou local.

Encontramos ainda a proposta de Husilos (2006), que considera três categorias de observatório: a) um local de documentação (armazenamento, classificação de informações e documentos); b) um local de análise de dados, considerado como ferramenta de apoio à tomada de decisões, a qual garante o reconhecimento, processamento e acesso à informação e o conhecimento de um determinado tema e; c) um espaço de informação, troca e interação que se distingue pela adaptação às tecnologias de informação e comunicação, permitindo recolher, tratar e divulgar informação, conhecer um tema e promover a reflexão em rede.

Neste trabalho, consideramos a definição de Antunes e Mangueira (2005), que afirmam que um observatório pode ser considerado como um modelo facilitador entre as universidades, 0 setor produtivo e o setor público, permitindo a identificação de demandas geradoras de emprego e renda, aumento da competitividade, identificação de novas tendências, por meio da gestão da informação e do conhecimento gerado.

\subsection{Observatório como uma ferramenta prospectiva}

Na década de 1990, Brisolla et al. (1996) já ressaltavam que responder pela necessidade de informações, nos níveis macros, médios e micros das atividades científicas e tecnológicas, é algo que pode ser executado por um observatório. Não somente para orientar decisões, mas também para incentivar a capacidade de respostas das necessidades em CT\&I. Um observatório tem capacidade de responder a essas necessidades, sem perder a perspectiva de integração com as dinâmicas de conhecimento e inovação.

Para Back (2016), os observatórios podem responder às demandas de informação estratégica, aos diversos setores da economia, motivados pela crescente incerteza e complexidade dos mercados nos quais as instituições estão inseridas. Eles possuem dois grandes propósitos: informações e resultados. Ou seja: investigar, caracterizar, avaliar e discutir informações relevantes na área; relatar os resultados para a sociedade e oferecer um espaço aberto para contribuições de agentes externos interessados que compartilham interesses mútuos (MARCIAL, 2009).

A principal vantagem de um observatório é a obtenção de uma visão ampla, integrando perspectivas regionais e locais, permitindo o desenvolvimento científico e tecnológico local sem perder de vista a perspectiva nacional. As informações geradas pelo observatório podem ser utilizadas pelos responsáveis pela formulação de políticas de CT\&l; pesquisadores e empresários veem suas condições econômica e sociais transformadas (BRISOLLA et al., 1996), 
De acordo com Marcial (2009), a dependência de uma instituição com o ambiente onde está inserida, a obriga a estar atenta a mudanças. No entanto nem a tecnologia nem as informações são suficientes se as alterações não são incorporadas à organização. O autor afirma que está se consolidando um novo modo de produção de conhecimento, no qual a identificação de problemas e as pesquisas para a sua solução são feitas através de uma interação entre especialistas e outros atores organizados em redes colaborativas, compartilhando conhecimentos e decisões, que é o objetivo de um observatório.

Em relação ao desenvolvimento tecnológico, o observatório contribui para: a) acompanhar ou monitorar as evoluções de determinados campos tecnológicos ou de pesquisa; b) funcionar como antenas ou radares para antecipar ideias, tendências e identificar questões e soluções; c) construir indicadores confiáveis de CT\&I de uma região ou país e d) conceber e produzir indicadores referentes às atividades científicas, tecnológicas e de inovação e permitir sua interpretação em termos de posicionamento do país ou da região. Implementar um observatório funcional e flexível, com uma equipe de pessoas altamente qualificadas, proporciona vantagens para todos os participantes. Mas a questão vai além de simplesmente criar uma entidade; deve-se avaliar a capacidade das instituições e da rede de parceiros para produzir suas próprias informações, utilizando-se de estatísticas e indicadores.

\section{MODELO DE OBSERVATÓRIO PARA ICTS}

O modelo proposto busca atender as questões trazidas pelas teorias utilizadas, os dados levantados nos estudos teóricos e de campo, relacionando e aproximando-os, para apoiar na concepção e na estruturação do observatório, cujo objetivo é identificar demandas de pesquisa e aproximar o setor produtivo da ICT.

Uma visão sistêmica do processo de inovação não deve ser confundida com a priorização das atividades de desenvolvimento tecnológico em detrimento da pesquisa científica. É necessário articular a produção científica com a produção tecnológica. Segundo Suzigan, Albuquerque e Cario (2011 , p. 9), "os institutos de pesquisa produzem conhecimento científico que é absorvido pelas empresas, e estas acumulam conhecimento tecnológico, fornecendo questões para a elaboração científica". Essa articulação e interação da produção científica com desenvolvimento tecnológico, oferta com demanda de conhecimento, pesquisa básica (também chamada de pesquisa fundamental) com pesquisa aplicada (que objetiva utilizar a informação disponível para criação de novas tecnologias e métodos) e o desenvolvimento de novos produtos e processos seriam, portanto, a chave de um sistema de inovação capaz de alavancar o desenvolvimento econômico dos países (DE NEGRI; CAVALCANTI, 2013).

É possível estruturar o observatório para analisar o ambiente externo e identificar as tendências para antecipar-se no desenvolvimento de inovações e mapear possíveis parceiros para desenvolvimentos em conjunto (PARREIRAS; ANTUNES, 2012). É preciso ainda, analisar a dimensão temporal (curto, médio e longo prazo), as variáveis a serem monitoradas (tendências futuras, riscos, investimentos em P\&D, dinâmica do desenvolvimento tecnológico) mercado, atores principais, abrangência territorial dos estudos, o público-alvo e os recursos necessários.

O modelo proposto neste artigo (figura 1) objetiva criar uma rede com a participação de atores públicos e privados para prospectar demandas de pesquisa científica e tecnológica que promovam o desenvolvimento e o aumento da competitividade e do setor. As principais finalidades do observatório são: a) identificar demandas da cadeia produtiva e alternativas para 
solucioná-las; b) ampliar a interação público-privada c) prospectar oportunidades em PD\&l; d) estimular a formalização de parcerias entre os atores; otimizar recursos humanos e financeiros para reduzir tempo na busca de soluções. A interação entre os atores públicos e privados e a equipe de pesquisa da ICT, permitirá troca de informação mútua e constante. Pressupõe-se, então, que as pesquisas a serem desenvolvidos em parceria público-privada atenderão as demandas presentes e futuras dos usuários.

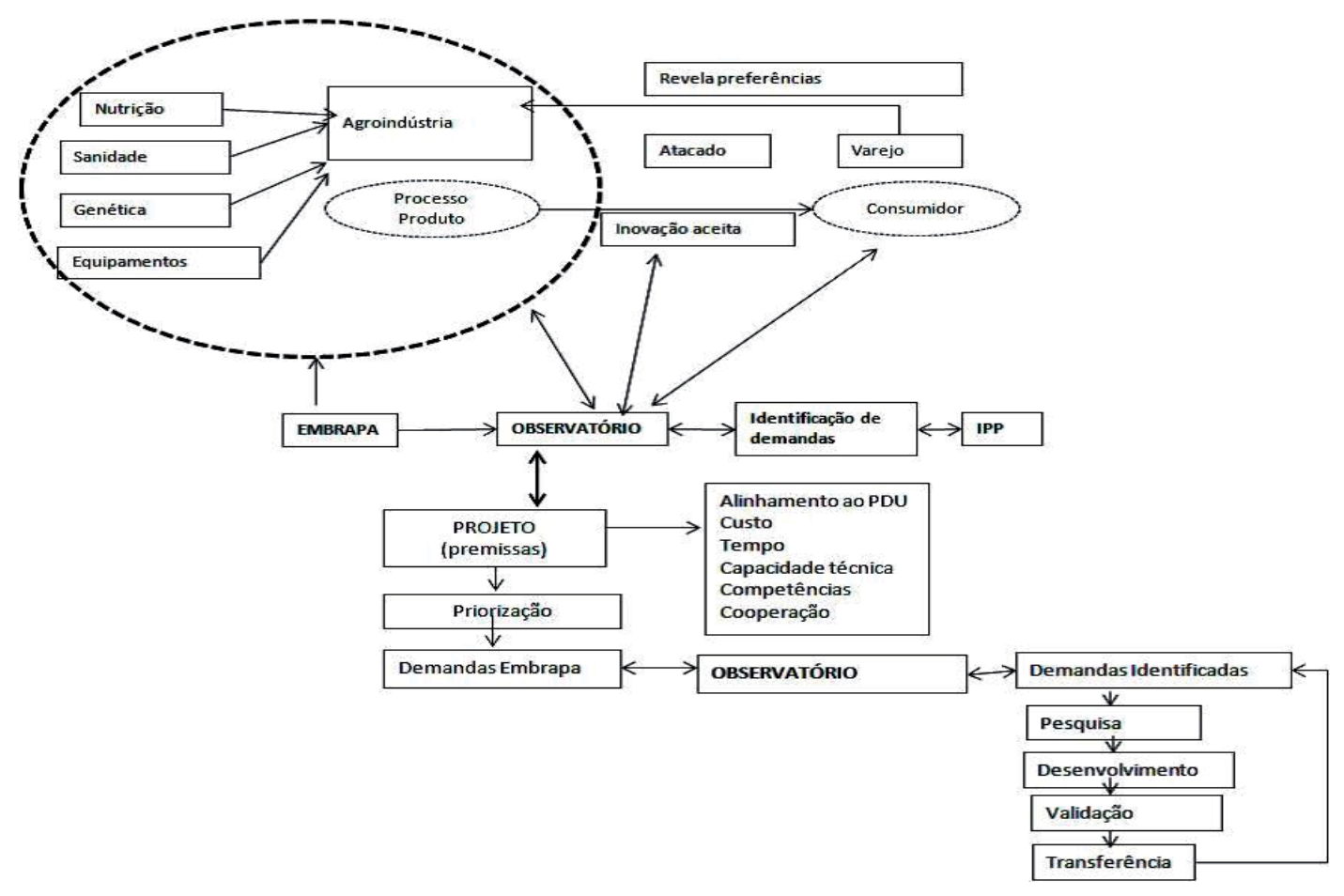

Figura 1 - Modelo de Observatório Proposto

Fonte: Autoria própria (2015).

Para a implantação do observatório, é necessário superar alguns desafios (PARREIRAS; ANTUNES, 2012): nem todos os pesquisadores conseguem quebrar paradigmas e pensar "fora da caixa" para a realização de estudos de prospectivos; alguns pesquisadores estão muito envolvidos em seus projetos de P\&D e podem enviesar os resultados do trabalho prospectivo, conduzindo-os para seus próprios temas de estudo; é indispensável o apoio dos tomadores de decisão na instituição.

\subsection{Forma de atuação do Observatório}

O modelo foi construído baseado nas características da Embrapa, como uma ICT e da cadeia produtiva de frangos de corte, todavia pode ser replicado para outras ICTs e cadeias.

A agroindústria, como processadora e distribuidora dos produtos no mercado, mantém uma estreita ligação com o consumidor, que revela suas preferências. Para atendê-las, muitas vezes, há necessidade de novas tecnologias advindas dos demais elos (nutrição, equipamento, genética e sanidade), considerados "fornecedores". Dessa forma, o observatório deve ser formado por representantes de todos os elos da cadeia, que, através de um processo interativo, identificarão as demandas atuais e futuras para cada elo e a possibilidade da formalização de parcerias para atendê-las. 
Em relação à participação da ICT nesse processo, propõe-se que as demandas identificadas no observatório sejam analisadas de acordo com os critérios de cada instituição (carteira de projeto, alinhamento ao plano estratégico, competências, custos, parcerias, etc.), para verificar a possibilidade de atendimento.

A interação entre participantes do observatório poderá ocorrer tanto por meio de uma plataforma on line para discussão, como em encontros periódicos anuais. Ainda que o modelo não contemple todos os aspectos, as principais características que devem estar presentes na busca pela prospecção de tendência, de acordo com os atores consultados (interatividade, prospecção, parcerias), foram incluídas e podem aprimorar o processo de prospecção e de desenvolvimento de tecnologias aplicadas.

Esse modelo propõe uma relação interativa entre os elos da cadeia, equipe técnica e direção da ICT. Seu uso implicará a adoção do modelo interativo de pesquisa e inovação, ou seja, o desenvolvimento de soluções para as demandas reais da cadeia, identificadas pelos usuários, torna os resultados mais efetivos e, consequentemente, dá maior legitimidade da ICT perante a sociedade. A operacionalização completa do modelo proposto não foi objeto deste estudo, porque cada ICT deverá optar pela forma de implantação que melhor Ihe convier, de acordo com as possibilidades e normativas que a regem.

O modelo proposto foi validado junto a 167 agentes dos elos de nutrição, genética, equipamentos, sanidade e agroindústrias, sendo aprovado por $96,77 \%$, que manifestaram interesse em participar de forma efetiva do observatório.

\section{CONCLUSÕES}

Com o acirramento da competição no contexto mundial, as instituições públicas e privadas precisam monitorar constantemente o ambiente externo para desenvolver práticas de planejamento e visão de futuro, que possibilitem direcionar o foco também para ações de longo prazo (PARREIRAS; ANTUNES, 2012). Uma forma estruturada de realizar essas atividades é por meio da implantação de um de observatório de tendências que contemple aspectos como: dimensões; variáveis; produtos do observatório; indicadores e público-alvo.

As informações geradas por um observatório podem ser utilizadas pelos responsáveis pela formulação de políticas públicas; por pesquisadores das ICTs; por empresários e pela própria sociedade para modificar suas condições econômica e sociais (BRISOLLA et al., 1996; ANTUNES; MANGUEIRA, 2005; CENTRO DE GESTÃO E ESTUDOS ESTRATÉGICOS [CGEE], 2006). Os observatórios são uma alternativa eficiente para responder às demandas estratégicas, uma vez que podem identificar problemas e formas de solucioná-los, por meio de uma interação entre especialistas e atores organizados em redes colaborativas (BACK, 2016; MARCIAL, 2009; BRISOLLA et al., 1996).

As ações de prospecção e identificação de oportunidades, considerando as tecnologias portadoras de futuro, os mercados, os aspectos sociais e oportunidades de parcerias público- privada, fortalecerão a interação entre a ICT e a cadeia. Dessa forma, o observatório proposto neste estudo poderá se constituir em um espaço de competência, com horizonte de longo prazo, que possibilitará subsidiar os processos de tomada de decisão sobre os temas e áreas prioritárias de pesquisa de uma ICT, baseados em evidências e em percepções do futuro. Ele oferece um ambiente para obtenção de consensos, identificação de oportunidade e parcerias, tornando-se um agente que fornece subsídios técnicos de alto nível e visões antecipadas do futuro para tomada de decisões estratégicas. 


\section{REFERÊNCIAS}

AMPARO, K. K. S.; RIBEIRO, M. C. O.; GUARIEIRO, L. L. N. Estudo de caso utilizando mapeamento de prospecção tecnológica como principal ferramenta de busca científica. Perspectivas em Ciência da Informação, Belo Horizonte, v. 17, n. 4, p. 195-209, out./dez. 2012.

ANTUNES, A. M. S.; MANGUEIRA, A. C. S. A importância do observatório de atividades industriais vis-a-vis tendências em ciência, tecnologia e inovação. Química Nova, São Paulo, v. 28, suplemento, p. S112-S118, nov./dez. 2005.

ASSOCIAÇÃO BRASILEIRA DE PROTEÍNA ANIMAL (ABPA). A avicultura brasileira. 2015. Disponível em: <http://www.ubabef.com.br/a_avicultura_brasileira/historia_da_avicultura_no_brasil>. Acesso em: 6 maio 2017.

.Relatório Anual 2016. Disponível em: <http://abpa-br.com.br/storage/files/versao_final_para_ envio_digital_1925a_final_abpa_relatorio_anual_2016_portugues_web1.pdf $>$. Acesso em: 17 fev. 2017.

AUDRETSCH, D. B.; LEYDEN, D. P.; LINK, A. N. Universities as research partners in publicly supported entrepreneurial firms. Economics of Innovation and New Technology, v. 21, n. 5-6, p. 529-45, 2012.

BACK, S. Modelo de observatório para apoio ao processo de inovação nas organizações: aplicação para as indústrias brasileiras de bens de capital. 2016. 376p. Tese (Doutorado em Ciência e Engenharia de Materiais)- Universidade Federal de Santa Catarina (UFSC), Florianópolis, SC, 2016.

BASSI, N. S. S; SILVA, C. L.; SANTOYO, A. Inovação, pesquisa e desenvolvimento na agroindústria avícola brasileira. Estudos Sociedade e Agricultura, Rio de Janeiro, v. 21, n. 2, p. 392-417, out. 2013/mar. 2014.

BASSI, N. S.; SILVA, C. L.; FIGUEIREDO, E. A. P. Technology transfer model proposal in public research institutions: the search for a more effective process in the broiler chain in Brazil. Journal of Technology Management \& Innovation, Santiago, Chile, v. 10, n. 4, p. 9-17, 2015.

BASSI, N. S. S. Proposta de um processo de transferência de tecnologia para as instituições públicas de pesquisa: o caso da Embrapa. 2015. 270p. Tese (Doutorado em Tecnologia)- Universidade Tecnológica Federal do Paraná (UTFPR), Curitiba, PR, 2015.

BOEHM, D. N.; HOGAN, T. Science-to-business collaborations: a science-to-business marketing perspective on scientific knowledge commercialization. Industrial Marketing Management, v. 42, n. 4, p. 564-79, maio 2013.

BRISOLLA, S. et al. Hacia la construcción de un Observatorio de Ciencia y Tecnología. Bogotá: Colciencias, 1996.

CAMPOS, Ana, L.S. Ciência, tecnologia e economia. In: PELAEZ, V.; SZMRECSÁNYI, T. (Org.). Economia da inovação tecnológica. São Paulo: Hucitec, 2006.

CANONGIA, C.; PEREIRA, M. N. F.; ANTUNES, A. M. S . Modelo de estratégia de prospecção de setores intensivos em P\&D: sinergias entre Inteligência Competitiva (IC), Gestão do Conhecimento (GC), e Foresight (F). BRAPCl, v. 7, n. 1, 2006. Disponível em: <http://www.brapci.inf.br/index.php/article/ view/0000003693/dabafa0e8a4031a013b529f008c2777b/>. Acesso em: 18 mar. 2017.

CENTRO DE GESTÃO E ESTUDOS ESTRATÉGICOS (CGEE). Observatório de Ciência, Tecnologia e Informação. Brasília: Centro de Gestão e Estudos Estratégicos, 2006. 83p.

CORDEIRO, L. P. A. Em busca de um modelo informacional evolutivo. In: ENCONTRO NACIONAL DE PESQUISA EM CIÊNCIA DA INFORMAÇÃO, 5. 2003, Belo Horizonte. Anais... Belo Horizonte: UFMG, 2003.

COSTA, L. S.; GARCIA, L. A. F.; BRENE, P. R. A. A indústria de frango de corte no mundo e no Brasil e a participação da indústria avícola paranaense neste complexo. Ciências Sociais em Perspectiva, v. 14, n. 27, p. 319-41, 2015.

DE LA VEGA, I. Tipología de Observatorios de Ciencia y Tecnología. Los casos de América Latina y Europa. Revista Española De Documentación Científica, v. 30, n. 4, p. 545-52, 2007. 
DE NEGRI, F.; CAVALCANTE, L. R. Sistemas de inovação e infraestrutura de pesquisa: considerações sobre o caso brasileiro. Radar - Tecnologia, Produção e Comércio Exterior, Brasília, n. 24, fev. 2013. Disponível em: <http://www.ipea.gov.br/radar/temas/infraestrutura/236-radar-n-24-sistemas-de-inovacao-einfraestrutura-de-pesquisa>. Acesso em: 18 mar. 2017.

DEVORE, P. W. Technology and science. In: ISRAEL, E. N.; WRIGHT, R. T. (Ed.). Conducting technical research. Mission Hills, CA: Glencoe, 1987.

DOSI, G. Technological paradigms and technological trajectories: a suggested interpretation of the determinants and directions of technical change. Research Policy, v. 11, n. 3, p. 147-62, jun. 1982.

FEDERAÇÃO DAS INDUSTRIAS DO PARANÁ (FIEP). O que é um Observatório? [s.d.]. Disponível em: <http:// www.fiepr.org.br/observatorios/FreeComponent2272content11361.shtml>. Acesso em: 16 fev. 2017.

FREEMAN, C. Economics of industrial innovation. Cambridge: MIT, 1982.

GODET, M.; DURANCE, P. A prospectiva estratégica para as empresas e os territórios. Paris: Dunod, 2011.

ENJUNTO, N. Razón de ser de los observatorios. Jornada Observando observatorios: ¿nuevos agentes en el tercer sector? 2010. Disponível em: <http://www.plataformavoluntariado.org/ARCHIVO/documentos/ recursos/observando-observatorios.-nuevos-agentes-en-el-tercer-sector.pdf>. Acesso em: 9 jun. 2017.

HUSILLOS, J. Círculo para la calidad de los servicios públicos de l'Hospitalet". La organización municipal y la adaptación de los servicios públicos. Inmigración y gobierno local. Experiencias y retos. In: SEMINARIO INMIGRACIÓN Y EUROPA, 4., Barcelona, España, 14-15 de diciembre 2006. Disponível em: <https://www. files.ethz.ch/isn/145683/Inmigraci\%C3\%B3n\%20y\%20Gobierno\%20local\%20experiencias\%20y\%20 retos_FULL\%20TEXT.pdf>. Acesso em: 8 fev. 2018.

JESUS JUNIOR, C.; PAULA, S. R. L.; ORMOND, J. G. P.; BRAGA, N. M. A cadeia da carne de frango: tensões, desafios e oportunidades. BNDES Setorial, Rio de Janeiro, n. 26, p. 191-232, set. 2007.

KLINE, S.; ROSEMBERG, N. An overview of innovation. In: LANDAU, R.; ROSEMBERG, N. The positive sum strategy. Washington, D.C.: National Academy Press, 1986.

LAVIE, D.; DRORI, I. Collaborating for knowledge creation and application: the case of nanotechnology research programs. Organization Science, v. 23, n. 3, p. 704-24, 2012.

MARCIAL, N. A. ¿Qué son los observatorios y cuáles son sus funciones? Innovación Educativa, v. 9, n. 47, p. 5-17, abr./jun. 2009. Disponível em: <http://www.redalyc.org/articulo.oa?id=179414895002>. Acesso em: 9 jun. 2017.

MARCONI, M. A.; LAKATOS, E. M. Técnicas de pesquisa. 6. ed. São Paulo: Atlas, 2007.

MARRA, R.; SOUZA, G. S.; ALVES; E. R. A. Papel da Embrapa no desenvolvimento do agronegócio. Revista de Economia e Agronegócio, Viçosa, MG, v. 11, n. 1, 2013. Disponível em: <http://www.revistarea.ufv.br/ index.php/rea/article/view/214/232>. Acesso em: 6 jun. 2017.

MARTIN, B. Foresight in science and technology. Technology Analysis \& Strategic Management, v. 7, n. 2, p. 139-78, 1995.

MAYERHOFF, Z. D. V. L. Uma análise sobre os Estudos de Prospecção Tecnológica. Cadernos de Prospecção, Salvador, BA, v. 1, n. p. 7-9, 2008.

NELSON, Richard, R. The changing institutional requirements for technological and economic catch up. Presentation at DRUID Summer Conference 2004: Industrial

dynamics, innovation and development. Copenhagen, Denmark, 2004.

Disponível em: <http://www2.druid.dk/conferences/viewabstract.php?id=2521\&cf=16>. Acesso em: 17 fev. 2017.

PARREIRAS, V. M. A.; ANTUNES, A. M. S. Aplicação de foresight e inteligência competitiva em um centro de P\&D empresarial por meio de um observatório de tendências: desafios e benefícios. Gestão \& Conexões, 
Vitória, ES, v. 1, n. 1, p. 55-73, jul./dez. 2012. Disponível em: <http://www.spell.org.br/documentos/ ver/41059/aplicacao-de-foresight-e-inteligencia-competiti--->.

PORTER, A. Technology futures analysis: toward integration of the field and new methods. Technological Forecasting \& Social Change, v. 71, n. 3, p. 287-303, mar. 2004.

QUINTELLA, C. M.; MEIRA, M.; GUIMARÃES, A. K.; TANAJURA, A. S.; DA SILVA, H. R. G. Prospecção tecnológica como uma ferramenta aplicada em ciência e tecnologia para se chegar à inovação. Revista Virtual de Química, Niterói, RJ, v. 3, n. 5, p. 406-15, 2011.

SANTOS, B. S. Introdução a uma ciência pós-moderna. Rio de Janeiro: Graal, 1989.

SANZ, L.; ANTÓN, F.; CABELLO, C. La prospectiva tecnológica, como herramienta para la política científica y tecnológica. Documento de trabalho 99-04. Instituto de Estudios Sociales Avanzados (CSIC). 2010.

SHAPIRA, A.; GOLDENBERG, M. Soft considerations in equipment selection of building construction projetcts. Journal of Construction Engineering and Management, v. 133, n. 10, p. 749-60, out. 2007.

SCHWARTZMAN, S. A pesquisa científica e o interesse público. Revista Brasileira de Inovação, Campinas, SP, v. 1, n. 2, p. 361-95, jul./dez. 2002.

SOH, P. H.; SUBRAMANIAN, A. M. When do firms benefit from university-industry R\&D collaborations? The implications of firm R\&D focus on scientific research and technological recombination? Journal of Business Venturing, v. 29, n. 6, p. 807-21, nov. 2014

SUBRAMANIAN, A. M. ; LIM, K.; SOH, P.-H. When birds of a feather don't flock together: different scientists and the roles they play in biotech R\&D alliances. Research Policy, v. 42, n.3, p. 595-612, abr. 2013.

SUZIGAN, W.; AlBUQUerquE, E. M.; CARIO, S. A. F. (Org.). Belo Horizonte: Autêntica, 2011. (Economia, Política e Sociedade, 464 p.).

TESTA, P. Indicadores científicos y tecnológicos en Venezuela: de las encuestas de potencial al observatorio de ciencia, tecnología e innovación. Cadernos del Cendes, ano 19, n. 51, p. 43-64, set./dez. 2002.

VASCONCELLOS, R. R. de. Barreiras e facilitadores na transferência de tecnologia para o setor espacial: estudo de caso de programas de parceria das agências espaciais do Brasil (AEB) e dos EUA (NASA). 2008. 469p. Tese (Doutorado em Engenharia) - Escola Politécnica da Universidade de São Paulo (USP), São Paulo, 2008.

VILHA, A. M. Gestão de inovação nas empresas. São Paulo: Agência Brasileira de Desenvolvimento Industrial - Prefeitura de Diadema- SINDIPLAST- SINDIBOR, 2010.

\section{Sobre os autores:}

Nádia Solange Schmidt: Bióloga, doutora em Tecnologia. Setor de prospecção e avaliação de tecnologias. Área de transferência de tecnologia. Embrapa Suínos e Aves. E-mail: nadia.schmidt@embrapa.br

Christian Luiz da Silva: Bolsista Produtividade CNPq. Coordenador Adjunto dos Programas Profissionais de pós-graduação da Capes na área de Planejamento Urbano e Regional e Demografia. Membro de assessoramento da área de administração e economia da Fundação Araucária. Professor Associado da UTFPR. Professor permanente do mestrado e doutorado do Programa de Pós-graduação de Tecnologia e Sociedade (PPGTE) e do Programa de Pós-graduação em Planejamento e Governança Pública (mestrado profissional). Professor do departamento de Gestão e Economia (DAGEE). Graduado em economia, mestre e doutor em engenharia de produção e pós-doutor em administração pela USP. E-mail: christiansilva@utfpr.edu.br 